\title{
Motivación por la lectura académica de futuros docentes
}

\author{
Inmaculada Clotilde Santos Díazi \\ Universidad de Málaga, Málaga, España \\ María Juárez Calvilloii \\ Universidad de Málaga, Málaga, España \\ Ester Trigo lbáñez ${ }^{\mathrm{iii}}$ \\ Universidad de Cádiz, Cádiz, España
}

\begin{abstract}
Resumen
La lectura juega un papel esencial en la formación universitaria como instrumento de reflexión crítica y de desarrollo de conocimientos especializados. Por ello, este trabajo pretende conocer la motivación por la lectura académica de 145 estudiantes de $4 .{ }^{\circ}$ del Grado de Educación Primaria de la Universidad de Cádiz durante el curso 2018/2019. Los datos se recabaron mediante un cuestionario sobre los hábitos lectores que incluía la Escala de Motivación por la Lectura Académica (MUÑOZ et al., 2012). Los resultados muestran que, aunque casi la mitad de los estudiantes afirma que les gusta leer, manifiestan no estar motivados por la lectura de textos académicos. Además, el componente mejor valorado de la Escala de Motivación por la Lectura Académica es la utilidad, frente al interés o la importancia. Estos hallazgos invitan a buscar estrategias de fomento de la lectura entre los universitarios de manera integral desde las diferentes áreas de conocimiento.
\end{abstract}

Palabras clave

Motivación. Lectura académica. Educación. Formación inicial. Profesorado.

\section{Motivação para a leitura acadêmica de futuros professores}

\begin{abstract}
Resumo
A leitura desempenha um papel essencial no ensino universitário como instrumento de reflexão crítica e desenvolvimento de conhecimentos especializados. Por esse motivo, este trabalho tem como objetivo conhecer a motivação para a leitura acadêmica de 145 alunos do $4^{\circ}$ do Ensino Fundamental da Universidade de Cádiz durante o ano letivo de 2018/2019. Os dados foram coletados por meio de um questionário sobre hábitos de leitura que incluiu a Escala de Motivação para Leitura Acadêmica (MUÑOZ et al., 2012). Os resultados mostram que, embora quase metade dos estudantes afirme gostar de ler, revelam que não estão motivados pela leitura de textos acadêmicos. Além disso, o componente com melhor classificação da Escala de Motivação para Leitura Acadêmica é a utilidade, antes do interesse ou da importância. Esses achados convidam à busca de estratégias para promover a leitura entre estudantes universitários de maneira abrangente a partir das diferentes áreas do conhecimento.
\end{abstract}

\section{Palavras-chave}

Motivação. Leitura acadêmica. Educação. Formação. Professores.

Educ. Form., Fortaleza, v. 6, n. 1, e3535, jan./abr. 2021

DOI: https://doi.org/10.25053/redufor.v6i1.3535

https://revistas.uece.br/index.php/redufor/index 


\title{
Motivation for the academic reading of future teachers
}

\begin{abstract}
Reading plays an essential role in university education as an instrument for critical reflection and the development of specialized knowledge. For this reason, this work aims to know the motivation for the academic reading of 145 students of 4th of the Primary Education Degree of the University of Cádiz during the 2018/2019 academic year. The data was collected through a questionnaire about reading habits that included the Motivation Scale for Academic Reading (MUÑOZ et al., 2012). The results show that, although almost half of the students affirm that they enjoy reading, they state that they are not motivated by reading academic texts. Furthermore, the best rated component of the Motivation Scale for Academic Reading is profit, versus interest or importance. These findings invite the search for strategies to promote reading among university students in a comprehensive way from the different areas of knowledge.
\end{abstract}

\section{Keywords}

Motivation. Academic reading. Education. Initial training. Teachers.

\section{Introducción}

La lectura ha sido y sigue siendo en la actualidad uno de los tópicos más estudiados en el panorama internacional. No en vano, existe una amplia variedad de revistas científicas dedicadas a esta actividad desde diversos puntos de vista: la alfabetización - en cuanto a su enseñanza, aprendizaje y dificultades -, la literacidad crítica, los hábitos lectores de los distintos grupos sociales, etc. $Y$ es que, como señala Lomas (2003), la lectura es la herramienta más valiosa para aprender y transformar el mundo y, como corroboran Ananias y Santos (2019), el acceso a la lectura y la escritura ha posibilitado el ascenso de unas clases sociales a otros estratos. Bajo estas ideas, la investigación en esta materia cobra una alta relevancia social y, a menudo, los medios de comunicación informan sobre sus principales hallazgos, lo que hace que la comunidad científica se acerque a la población en general.

Desde nuestro punto de vista, los maestros se erigen como uno de los principales agentes responsables a la hora de desarrollar el hábito lector, así como la competencia lectora y literaria de las futuras generaciones. Por ello, en este trabajo realizaremos un breve recorrido por las investigaciones que, en los últimos años, han tomado como referencia los docentes en formación y estudiado su relación con la lectura (ELCHE; YUBERO, 2019). En este ámbito, son frecuentes los estudios que se

Educ. Form., Fortaleza, v. 6, n. 1, e3535, jan./abr. 2021

DOI: https://doi.org/10.25053/redufor.v6i1.3535

https://revistas.uece.br/index.php/redufor/index 
aproximan a los consumos literarios (DAISEY, 2009; GRANADO; PUIG, 2014; MUNITA, 2014, 2018, 2019; PARRADO; ROMERO; TRIGO, 2018) o que recogen las creencias de este grupo poblacional (ÁLVAREZ-ÁLVAREZ, 2018; ÁLVAREZ-ÁLVAREZ; DIEGOMANTECÓN, 2019, BIRELLO; GIL, 2014; CASTILLO-FADIĆ; SOLOGUREN-INSÚA, 2020; JUÁREZ, 2019; PÉREZ-PEITX, 2016) y sus preferencias de formato al afrontar la lectura académica (DOMÍNGUEZ-PÉREZ; PÉREZ-RUL, 2009; PARODI et al., 2019).

A través de estas investigaciones, se ha puesto de manifiesto la necesidad de indagar sobre la competencia lectora de los futuros docentes (FELIPE; BARRIOS, 2017; FELIPE; VILLANUEVA, 2018), pues se ha comprobado que los estudiantes no siempre cuentan con las habilidades lectoras que les habilitan para disfrutar de los textos literarios y académicos y, por tanto, no conectan emocionalmente con esta actividad (JIMÉNEZ-PÉREZ, 2018).

De acuerdo con estos planteamientos, Peredo (2001) ha recogido las habilidades lectoras que deberían poseer los estudiantes universitarios. Entre ellas destaca: el poder de síntesis, la capacidad para resumir, la comprensión y discriminación hacia la literatura científica, la capacidad de criticar y reflexionar a partir del diálogo, la confrontación ideológica, la sensibilidad literaria y, por último, la síntesis. Así, podemos afirmar que, en el ámbito universitario, la relación entre docentes y estudiantes con la lectura académica suele estar acompañada del concepto de frustración, pues, por un lado, los docentes encuentran que sus estudiantes bien no se interesan por las lecturas propuestas, bien no las comprenden. Por su parte, los estudiantes suelen abordar las lecturas académicas como una obligación para ir superando las distintas asignaturas (CARO, 2015).

Unido a esto, la imagen que reflejan los futuros docentes en cuanto a la lectura no difiere demasiado de su nivel de desempeño en escritura académica (JARPA, 2019; JARPA; BECERRA, 2019; ROMERO; JIMÉNEZ, 2015; TRIGO; NÚÑEZ, 2018; YOON; RÖMER, 2020). Así, resulta necesario trazar estrategias, en el marco de la institución universitaria, que desarrollen la competencia lectora y potencien la escritura reflexiva a través de la coordinación docente (BOLARÍN; MORENO, 2015; ROMERO; SALVADOR; TRIGO, 2014; SÁNCHEZ-JIMÉNEZ; GALIANO, 2019). Según Carlino (2005), este sería el mayor problema que enfrentan los estudiantes: hallarse frente a una nueva cultura escrita que exige a sus lectores, pero que entrega pocas herramientas.

Educ. Form., Fortaleza, v. 6, n. 1, e3535, jan./abr. 2021 
Sin embargo, consideramos que los estudiantes que leen con aprovechamiento las lecturas específicas en su formación como docentes están más capacitados para observar y describir adecuadamente la realidad de aula desde un enfoque pedagógico-reflexivo (BRANDT; HOBOLD, 2019; JARPA, 2019; PLAZAOLA; RUIZ; IRIONDO, 2018; ROMERO; JIMÉNEZ, 2014).

Como se aprecia, la motivación por la lectura académica no es una faceta aún muy estudiada. Tan solo conocemos los trabajos de Muñoz et al. $(2012,2016)$, realizados en Chile, donde se ha prestado atención a esta actividad tomando como referencia a fututos docentes y concluyendo que gran parte del alumnado se encuentra sin herramientas acerca de cómo abordar sus lecturas. En el contexto español, no nos constan investigaciones realizadas en esa línea. Así, pretendemos cubrir ese vacío acercándonos a la motivación de los docentes gaditanos en formación hacia la lectura académica.

Así, este estudio pretende, como objetivo general, determinar la motivación que los estudiantes del último curso del grado en Educación Primaria de la Universidad de Cádiz tienen por la lectura académica en sus dimensiones: expectativa, importancia, interés, utilidad y costo, con el fin de encontrar las áreas susceptibles de mejora. En este sentido, nos marcamos dos objetivos específicos:

- Conocer la valoración del futuro profesorado de Educación Primaria con respecto a diferentes afirmaciones sobre la lectura académica.

- Analizar la relación existente entre el tipo de mención - o especialidad - que cursan, con la importancia dada a la lectura de textos académicos.

\section{Metodología}

Este estudio se enmarca en el paradigma cuantitativo al ofrecer una visión estadística descriptiva del comportamiento de una población (SÁEZ, 2017). Sin embargo, los resultados no pretenden generalizarse, sino que ayudarán a comprender con mayor profundidad el contexto estudiado - en nuestro caso, la Universidad de Cádiz - y detectar los puntos mejorables para ofrecer soluciones efectivas. Se trata de una investigación centrada en contextos (MENDOZA, 2011). 


\subsection{Participantes}

La muestra está formada por 145 estudiantes de tres grupos diferentes del $4 . .0$ curso del Grado de Educación Primaria de la Universidad de Cádiz durante el curso 2018/2019 ( $n=48$ hombres y $n=106$ mujeres). Tan solo hay cuatro informantes de una nacionalidad diferente a la española (austríaca, italiana, keniana y suiza). La edad de los informantes oscila entre los 21 y los 44 años y la media se sitúa en los 22,84 años.

La Tabla 1 recoge la distribución porcentual del total de la muestra y la frecuencia según la mención cursada - es decir, el tipo de especialización dentro del Grado en Educación Primaria - y el sexo. Las menciones más elegidas de mayor a menor número de informantes son: Educación Física $(n=41)$, Educación Especial $(n=41)$, Enseñanza a través de proyectos integrados $(n=23)$, Lengua Extranjera AICLE $(n=33)$, Educación Musical ( $n=12)$ y, en último lugar, los estudiantes sin mención $(n=4)$.

En cuanto al sexo de los informantes, destaca la amplia mayoría de mujeres, 106, que suponen un $68,8 \%$ frente a 48 hombres, que conforman el $31,2 \%$ del total. Las mujeres predominan en todas las menciones, excepto en la de Educación Física, donde este dato se invierte. Este hecho se debe a que tradicionalmente se ha adscrito la docencia al ámbito femenino. Es por ello por lo que, siguiendo estos patrones de género tradicionales, la actividad física se ve asociada a los hombres.

Tabla 1 - Descripción de la muestra según las variables estudiadas

\begin{tabular}{lccccccc}
\multicolumn{1}{c}{ Mención } & \multicolumn{2}{c}{ Hombres } & \multicolumn{2}{c}{ Mujeres } & \multicolumn{2}{c}{ Total } \\
Educación Física & $\mathbf{n}$ & $\%$ & $\mathbf{n}$ & $\%$ & $\mathbf{n}$ & $\%$ \\
Educación Especial & 25 & 16,23 & 16 & 10,39 & 41 & 26,62 \\
Enseñanza a través de proyectos & 6 & 3,90 & 35 & 22,73 & 41 & 26,62 \\
integrados & 8 & 5,19 & 15 & 9,74 & 23 & 14,94 \\
Lengua Extranjera - AICLE & 4 & 2,60 & 29 & 18,83 & 33 & 21,43 \\
Educación Musical & 4 & 2,60 & 8 & 5,19 & 12 & 7,79 \\
Ninguna & 1 & 0,01 & 3 & 0,02 & 4 & 0,03 \\
Total & 48 & 31,17 & 106 & 68,83 & 154 & 100 \\
\hline
\end{tabular}

Fuente: Elaboración propia (2020).

\subsection{Instrumento}

Los datos se han obtenido mediante la utilización de una versión revisada de la Escala de Motivación por la Lectura Académica (EMLA) validada estadísticamente 
(MUÑOZ et al., 2012) ${ }^{1}$. A ella se han añadido ítems de naturaleza cualitativa enfocados a perfilar a los futuros maestros como sujetos lectores didácticos (DELBRAYELLE; DUSZYNSKI, 2007; MUNITA, 2018, 2019) y a explorar sus creencias en torno a la lectura y la educación literaria (ÁLVAREZ-ÁLVAREZ; DIEGO-MANTECÓN, 2019; JUÁREZ, 2019) e ítems relacionados con la recepción de contenido audiovisual, lo que posibilitará, en una futura investigación, correlacionar el léxico disponible de los informantes en español, inglés y francés con sus consumos mediáticos (SANTOS-DÍAZ, 2017; SANTOS-DÍAZ; TRIGO; ROMERO, 2020).

El cuestionario completo ha sido validado por un grupo de investigadores, integrantes del grupo de investigación HUM-1041 Investigación e innovación educativa en Didáctica de la Lengua y la Literatura, especialistas en Didáctica de la Lengua y la Literatura y en Disponibilidad Léxica. Además, se realizó una prueba piloto, cuestión que permitió realizar los ajustes necesarios para asegurar su validez y confiabilidad.

Así, la versión final contiene cuatro secciones: Información personal y académica, a través de la cual se pretende conocer las características socioeconómicas de los participantes, además de profundizar en los estudios de procedencia y la vía de acceso al Grado de Magisterio en Educación Primaria; Hábitos lectores y educación literaria, que permitirá describir al informante como sujeto lector literario; Motivación con la lectura académica, que favorecerá el conocimiento de la relación de los futuros maestros con los textos científicos propios de la epistemología de los estudios cursados; $\mathrm{y}$, en último lugar, Recepción de léxico audiovisual, donde se indaga acerca de la exposición a las lenguas extranjeras de los informantes en su tiempo libre: música, películas, series de televisión, subtítulos, etc.

En concreto, en esta investigación se analiza una pregunta sobre el gusto por la lectura, otra sobre la motivación por la lectura según el tipo de texto y la EMLA. Esta escala está compuesta por 27 ítems que se dividen en cinco componentes 0 dimensiones: expectativa, interés, importancia, utilidad y costo. Los informantes respondieron indicando su grado de conformidad con estas afirmaciones siguiendo una escala de Likert. Esta escala sirve para medir el grado de satisfacción de los encuestados con respecto a distintos enunciados. Para su configuración, se ha seguido el criterio de

1 Agradecemos al Proyecto Fondecyt Regular n. 1170779 que nos facilitara su cuestionario para que pudiéramos adaptarlo a nuestra investigación.

Educ. Form., Fortaleza, v. 6, n. 1, e3535, jan./abr. 2021

DOI: https://doi.org/10.25053/redufor.v6i1.3535

https://revistas.uece.br/index.php/redufor/index 
Matas (2018, p. 2), utilizando cinco valores: "[...] esta escala incluía un punto medio neutral, así como puntos a izquierda y derecha, originalmente de desacuerdo y de acuerdo, con opciones de respuesta numéricas de 1 a 5". Esta decisión hace que los resultados no puedan compararse en términos exactos con los de otros estudios (MUÑOZ et al., 2012, 2016), pues estos autores siguen una escala que oscila entre 1 y 6 .

\subsection{Procedimiento}

El cuestionario se aplicó en formato papel y, posteriormente, los datos se codificaron en una matriz que permitió su procesamiento con el paquete estadístico SPSS, versión 23. Los análisis realizados se corresponden con una estadística descriptiva.

\section{Resultados}

\subsection{Gusto por la lectura}

Al analizar la pregunta "¿Te gusta leer?", hemos organizado las distintas respuestas en dos grupos: positivas y negativas. Los valores eran originalmente nada, muy poco, algo, bastante y mucho. Hemos otorgado un valor negativo a las dos primeras opciones y un valor positivo a las tres restantes. Así - vide Gráfico 1 -, es destacable que, a pesar de que un 49,35 \% contestó negativamente - lo que nos da una clara visión de que la mitad de los encuestados no disfrutan de la práctica lectora -, la otra mitad $(50,65 \%)$ respondió con mayor positividad. Teniendo en cuenta que los encuestados son estudiantes de último curso de Grado Universitario de Magisterio, el alto porcentaje de respuestas negativas obtenidas supone un dato bastante desfavorable de cara al ejercicio de su futura profesión (LARRAÑAGA; YUBERO, 2008). Además, resulta preocupante que se trate de una imagen repetida en numerosas investigaciones realizadas con futuros docentes (GRANADO; PUIG, 2014; MUNITA, 2014; PARRADO; ROMERO; TRIGO, 2018; ELCHE; YUBERO, 2019, JUÁREZ, 2019, entre otras).

Educ. Form., Fortaleza, v. 6, n. 1, e3535, jan./abr. 2021 


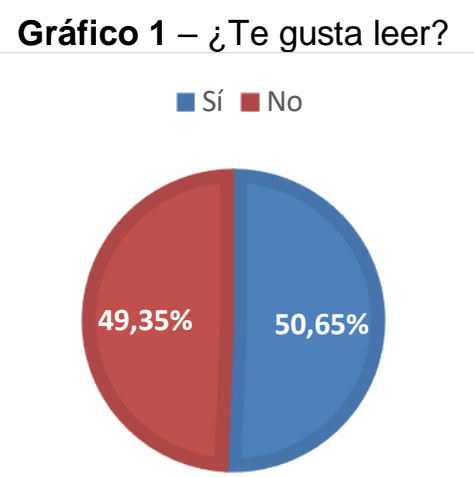

Fuente: Elaboración propia (2020).

\subsection{Motivación por la lectura según tipo de texto}

En cuanto a la motivación específica por la lectura de cada tipo de texto explorado: académicos, literarios, informativos y mensajes en redes sociales - vide Gráfico 2 -, observamos que los textos que los informantes leen con mayor grado de motivación (5) son los mensajes en redes sociales (33,12\%), seguidos de los textos literarios $(29,87 \%)$, los informativos (15,58\%) y, en último lugar, los académicos (1,95\%). Es obvio que, cuando nos referimos a motivación, los encuestados se sienten más atraídos por leer textos que les suponen más disfrute y evasión frente a textos informativos o académicos, como muestran estudios en los que los textos académicos se quedan atrás en la clasificación (ÁLVAREZ-RAMOS; HEREDIA; ROMERO, 2019; JIMÉNEZ-PÉREZ, 2018; MUÑOZ et al., 2018). Así pues, en el otro extremo, estos resultados son proporcionales, por lo que consecuentemente los textos que han valorado con menor grado de motivación (1) son los siguientes: textos académicos $(42,86 \%)$, textos literarios $(11,69 \%)$, textos informativos $(6,49 \%)$ y mensajes en redes sociales $(4,55 \%)$.

De la misma manera, y como hemos comentado anteriormente, estos datos suponen una visión bastante negativa de cómo se acercan los futuros docentes a la lectura. Podemos pensar que los encuestados ven la lectura de textos académicos como herramienta para superar asignaturas de forma obligatoria más que como método indispensable para su propia formación, cuestión que concuerda con estudios precedentes (APPLEGATE, A.; APPLEGATE, M., 2004; APPLEGATE et al., 2014; CARO, 2015). 
Gráfico 2 - Motivación por la lectura de textos académicos, literarios, informativos y mensajes en redes sociales

\begin{tabular}{|c|c|c|c|c|}
\hline \multirow{9}{*}{$\begin{array}{r}100 \% \\
90 \% \\
80 \% \\
70 \% \\
60 \% \\
50 \% \\
40 \% \\
30 \% \\
20 \% \\
10 \% \\
0 \%\end{array}$} & & & & \\
\hline & & & & \\
\hline & & & & \\
\hline & & & & \\
\hline & & & & \\
\hline & & & & \\
\hline & & & & \\
\hline & & & & \\
\hline & Textos académicos & Textos literarios & Textos informativos & $\begin{array}{c}\text { Mensajes en redes } \\
\text { sociales }\end{array}$ \\
\hline$\square, 0$ & 1,95 & 29,87 & 15,58 & 33,12 \\
\hline 4,0 & 6,49 & 30,52 & 25,97 & 38,31 \\
\hline 3,0 & 18,18 & 16,23 & 28,57 & 12,99 \\
\hline 2,0 & 30,52 & 11,69 & 23,38 & 11,04 \\
\hline$\square 1,0$ & 42,86 & 11,69 & 6,49 & 4,55 \\
\hline
\end{tabular}

Fuente: Elaboración propia (2020).

\subsection{Resultados generales de la EMLA}

Como se señala en la investigación de Muñoz et al. (2016), los 27 ítems que componen la EMLA - vide Tabla 1 del Anexo - se reagrupan atendiendo a su contenido. De esta forma, siguiendo los presupuestos de Wigfield y Eccles (2000), se establecen cinco componentes:

a) Expectativa, donde se indaga sobre la relación entre la lectura académica y el éxito escolar (ítems 5, 7, 13,16 y 21) (ROMERO; TRIGO, 2015).

b) Importancia, a través del cual se puede reflexionar sobre el grado de importancia que otorgan los informantes al hecho de realizar bien sus lecturas específicas (ítems 2, 7, 12, 18, 23 y 25) (DECl; RYAN, 1985).

c) Interés, desde el que se da cuenta del disfrute que proporciona a los informantes el hecho de asumir la tarea de leer textos académicos (ítems 3 , 10, 15, 19, 22, 24 y 26) (SILVIA, 2006).

d) Utilidad, que, en términos instrumentales, se refiere a la medida en la que los informantes perciben que la lectura académica es relevante para su futuro desempeño profesional (ítems 1, 6, 11, 14 y 17) (TERCANLIOGLU, 2001).

e) Costo, donde se da cuenta del esfuerzo que requiere para los informantes abordar la lectura especializada y el desgaste emocional que les supone (ítems 4, 8, 20 y 27) (ORIOL-GRANADO et al., 2017). 
En primera instancia, ofrecemos una panorámica tomando como referencia los valores medios de cada componente. En segunda instancia, analizamos la media otorgada en los distintos componentes. Así, observamos que, en líneas generales - vide Gráfico 3 -, el componente mejor valorado por los informantes es utilidad, con una media de 3,54; seguido de costo y expectativa, 3,39 y 3,35, respectivamente; interés, cuya media se sitúa en 2,95; y, por último, importancia, que registra un valor medio de 2,73. De esto se desprende que los estudiantes consideran la lectura académica como un elemento que les ayudará a superar sus estudios universitarios. Sin embargo, no les resulta demasiado interesante afrontar la lectura de este tipo de textos ni se preocupan en exceso por profundizar en ellas.

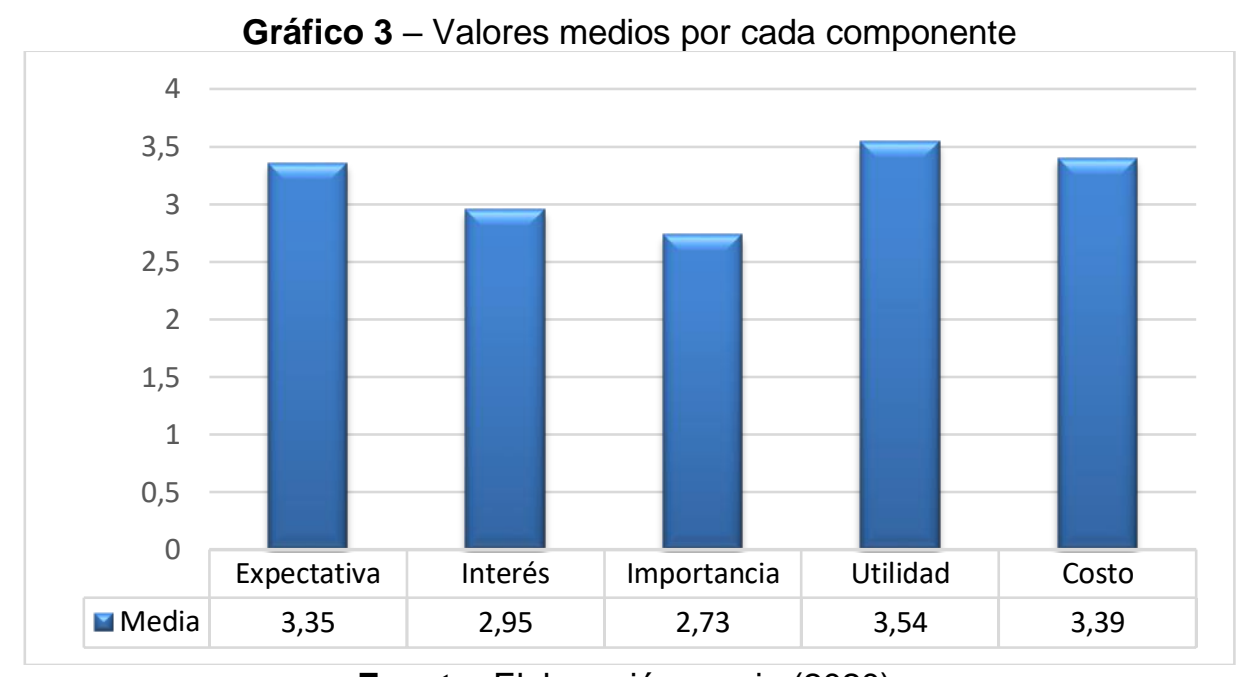

Fuente: Elaboración propia (2020).

Si analizamos detenidamente los ítems que componen la EMLA, lo primero que llama la atención en su autoevaluación y expectativa como lectores de textos académicos es la visión que los informantes tienen sobre sí mismos es bastante positiva. Así, atendiendo al ítem 5: "Considero que escojo bien los textos académicos que me ayudan a realizar mis trabajos", vemos que se sienten con bastante confianza a la hora de discernir qué textos son de mayor importancia (un $44,81 \%$ responde con un 3 sobre 5); de distinguir las ideas centrales, como se recoge en el ítem 13: "Soy capaz de distinguir las ideas centrales de las complementarias en los textos académicos" (el $33,77 \%$ otorga el valor 4 sobre 5 ); en la comprensión de los documentos que deben leer, reflejada en el ítem 16: "A pesar de que algunos textos académicos son complejos, 
soy capaz de comprenderlos si me esfuerzo" (un 40,91\% de los informantes se sitúa en un 4 sobre 5); o la capacidad para afrontar la lectura académica, explorada en el ítem 21 "Estoy capacitado para leer la mayoría de los textos académicos en mi área disciplinar" (el 38,31\% señala un 4 sobre 5 ).

Sin embargo, a pesar de que los estudiantes son conscientes de la importancia de la lectura de textos académicos formativos, el ítem 7, "Para mí, la bibliografía mínima es importante para comprender la materia de los cursos", registra valores más desfavorables (el 18,83\% otorga el valor 1 frente al 3,90\% registrado en el valor 5 ). Unido a esto, como se refleja en el ítem 10, "Me interesa leer material más allá de lo requerido por el curso", los informantes no suelen profundizar con lecturas académicas más allá de las obligatorias, generalmente facilitadas por los docentes (el 14,94 \% otorga el valor 1 frente a 6,99\% que le otorga un valor 5 ).

Al fijarnos en los ítems 3, "Me gusta leer textos académicos relacionados con mi carrera"; 4, "Soy capaz de dejar otros intereses de lado y comprometerme hasta terminar de manera debida la lectura de un texto", y 8, "El tiempo que utilizo en leer textos académicos implica dejar de hacer otras cosas", comprobamos que gran parte de los encuestados disfruta de la lectura de textos académicos relacionados con su grado universitario y se siente muy comprometido con esta actividad. Así, en el ítem 3, el $60,39 \%$ de los informantes otorga una puntuación 3 o 4 sobre 5 . En el ítem 4, este porcentaje asciende al 50,64 y en el 8 al 55,19. Sin embargo, parecen contradecirse en algunos aspectos, pues, aunque reconocen la lectura académica como prioritaria frente a otras tareas o actividades, no muchos de ellos confiesan dedicar tiempo a resolver dudas en otras fuentes, como refleja el ítem 22, "Cuando tengo dudas después de clases, leo otras fuentes", donde tan solo un $25,97 \%$ otorga puntuaciones entre 4 y 5 .

Estas contradicciones también quedan manifiestas en el componente importancia, ya que, aunque gran parte de los encuestados considera que es importante leer y comprender la bibliografía mínima recomendable (ítems 2, "Es importante leer toda la bibliografía mínima de los cursos", 7, "Para mí, la bibliografía mínima es importante para comprender la materia de los cursos", 12, "Para mí, la bibliografía mínima es importante para complementar la materia de los cursos", y 23, "Para mí, es importante comprender el material bibliográfico de los cursos porque me permite dominar ciertos temas"), un gran número de encuestados confiesa no leer esta bibliografía previamente a 
la clase correspondiente, como queda patente en el ítem 25, "Para mí, es importante tener leída la bibliografía antes de la clase (cuando corresponde)". Así, los porcentajes más altos coinciden con las respuestas con menor conformidad con respecto a esta afirmación: un 24,68\% responde con un 1 y 25,32\% con un 2. En este sentido, podríamos afirmar que, aunque son conscientes de la importancia de la comprensión lectora, no desempeñan su labor académica de forma coherente.

En cuanto al componente utilidad, en el ítem 1, "La lectura de textos académicos es útil para mi formación profesional', vemos que la mayoría de los informantes comprende la utilidad de leer textos académicos para su formación, pues el 61,69 \% le otorga puntuaciones situadas entre 4 y 5 . De la misma forma, son conscientes de la importancia de la comprensión lectora y responden con coherencia en cuanto a la utilidad de estos. Por ello, en el ítem 6, "Considero de gran utilidad entender los textos académicos que se me asignan", un 32,47\% responde con una conformidad de 4 sobre 5 , un valor y porcentajes altos, al igual que en el ítem 11, "Considero útil la lectura de textos académicos para desarrollar ciertas competencias profesionales", donde un $37,01 \%$ responde también con 4 sobre 5 . Así, podemos apreciar que los estudiantes consideran de gran provecho y beneficio estos textos académicos que se les asignan, ya no solo para su formación académica, sino también para el desarrollo de sus competencias profesionales.

Atendiendo al costo, los encuestados afirman dedicar el tiempo necesario a las tareas de lectura para terminarlas de manera adecuada. Esta idea se ve reflejada en el ítem 20, "Cuando debo realizar una lectura académica, le dedico todo el tiempo necesario hasta terminarla de manera adecuada", donde un 36,36 \% responde con un 4 . Y siguiendo con esa visión positiva de sí mismos como estudiantes, también consideran, en su mayoría, que, si su comprensión escrita no es suficiente, invierten más tiempo para lograr realizar la actividad con éxito. Esto puede verse en el ítem 27, "Si mi comprensión del texto es insuficiente, soy capaz de invertir más tiempo en su lectura", donde un $31,17 \%$ responde con un 4 , es decir: se sienten altamente satisfechos y responsables a la hora de comprometerse con su comprensión escrita. 


\section{Consideraciones finales}

En este trabajo hemos tratado de conocer a los futuros maestros de Educación Primaria que están a punto de terminar su formación en la Universidad de Cádiz durante el curso 2018/2019. Hemos tenido la oportunidad de recoger los datos pertinentes con la EMLA (MUÑOZ et al., 2012) mediante un cuestionario que incluía otras preguntas de tipo sociológico y relacionadas con los hábitos lectores.

Al analizar los distintos componentes - expectativa, interés, importancia, utilidad y costo -, hemos podido descubrir que el alumnado encuestado no responde con la motivación adecuada en ningún aspecto, aunque un alto porcentaje de los encuestados afirma que le gusta leer. Esta aparente contradicción en los resultados puede deberse a que los encuestados optan más por dar una respuesta políticamente correcta que honesta y que la percepción acerca de su propio trabajo está idealizada.

Estas consideraciones nos hacen reflexionar sobre la posibilidad, en una futura investigación, de contrastar nuestros hallazgos con los resultados académicos de los estudiantes. Una vez realizado este contraste, deberíamos realizar una investigación cualitativa, organizando foros de discusión donde compartir los resultados obtenidos en la presente investigación para analizar la voz del equipo decanal, los docentes de las distintas menciones y los estudiantes. Esto nos ayudaría a configurar una imagen más certera del fenómeno estudiado y serviría para trazar estrategias verdaderamente efectivas, como acercarse a la lectura desde una perspectiva lúdica y digital (BOA, 2019).

Los resultados obtenidos abren líneas prospectivas en las que se fomente entre las diferentes áreas un trabajo de coordinación horizontal y vertical de manera íntegra (BOLARÍN; MORENO, 2015; ROMERO; SALVADOR; TRIGO, 2014; SÁNCHEZ-JIMÉNEZ; GALIANO, 2019) e incluso trascendiendo los límites de la universidad y fijando la mirada hacia la escuela, lugar donde los estudiantes desempeñarán su labor profesional, pues uno de los compromisos de la formación inicial debe centrarse en desarrollar la capacidad pedagógico-reflexiva de los futuros docentes (BRANDT; HOBOLD, 2019; JARPA, 2019; PLAZAOLA; RUIZ; IRIONDO, 2018; ROMERO; JIMÉNEZ, 2014).

Como en estudios precedentes (MUÑOZ et al., 2012), se reconoce ampliamente que no basta con "saber leer", sino que es necesario querer entrar en diálogo con la 
literatura académica, puesto que es allí donde el estudiante universitario se juega gran parte de la adquisición y actualización de conocimientos, marcando la diferencia entre un conocimiento superficial y el conocimiento profundo de un tema.

Si bien los resultados de esta investigación no pretenden ser generalizables, parecen atisbarse coincidencias con otros contextos, por lo que resultaría muy enriquecedor trazar líneas de acercamiento para trabajar en soluciones conjuntas, aunando esfuerzos que ayuden a dignificar la profesión docente.

\section{Referencias}

ÁLVAREZ-ÁLVAREZ, C. La formación inicial de los docentes en conferencias: una exploración basada en historias de vida. In: RUIZ-BEJARANO, A. M. (coord.). Educación, literacidades y ciudadanía: líneas actuales de debate. Alicante: Uno, 2018. p. 61-72.

ÁLVAREZ-ÁLVAREZ, C.; DIEGO-MANTECÓN, J. M. ¿Cómo describe, analiza y valora a los futuros maestros de su educación? Revista Complutense de Educación, Madrid, v. 30, n. 4, p. 1083-1096, 2019.

ÁLVAREZ-RAMOS, E.; HEREDIA, H.; ROMERO, M. F. Generación Z y las redes sociales. Una visión de los adolescentes en españa. Revista Espacios, Caracas, v. 40, n. 20, p. 9-21, 2019.

ANANIAS, M.; SANTOS, L. R. "Quando o homem sabe ler, escrever e contar, pode, por sua própria individualidade, desenvolver-se e esclarecer-se": a escolarização de crianças pobres na província da Parahyba do Norte (1855-1866). Educação \& Formação, Fortaleza, v. 4, n. 1, p. 66-80, 2019.

APPLEGATE, A. J.; APPLEGATE, M. D. The Petter effect: reading, habits and attitudes of preservice teachers. The Reading Teacher, v. 57, n. 6, p. 554-563, 2004.

APPLEGATE, A. J. et al. The Peter effect revisited: reading habits and attitudes of college students. Literacy Research and Instruction, v. 53, n. 3, p. 188-204, 2014.

BIRELLO, M.; GIL, M. R. Aprenderá sobre la composición escrita y la gramática de los alumnos de jardín de infantes y educación primaria. Tejuelo: Didáctica de la Lengua y la Literatura, Extremadura, v. 10, p. 11-26, 2014.

BOA, P. Internet memes: classroom perspectives in the context of digital cultures. Educação \& Formação, Fortaleza, v. 4, n. 3, p. 51-66, 2019. 
BOLARÍN, M. J.; MORENO, M. Á. El coordinador docente de la universidad: derechos y problemas de Bolonia. Profesorado: revisión del currículum y formación del profesorado, Granada, v. 19, n. 2, p. 319-332, 2015.

BRANDT, A.; HOBOLD, M. A prática como componente curricular na disciplina Pesquisa e Processos Educativos do curso de Pedagogia: um diferencial na relação entre pesquisa, teoria e prática. Educação \& Formação, Fortaleza, v. 4, n. 2, p. 142-160, 2019.

CARLINO, P. Escribir, leer y aprender en la universidad. Buenos Aires: Fondo de Cultura Económica, 2005.

CARO, S. Lecturas académicas. Estrategias didácticas para el enfoque de la enseñanza en la universidad. Reflexión Académica en Diseño y Comunicación, Palermo, v. 25, p. 96-99, 2015.

CASTILLO-FADIĆ, M. N.; SOLOGUREN-INSÚA, E. Léxico frecuente, riqueza léxica y estereotipos sobre la lectura de profesores en formación. Logos: Revista de Lingüística, Filosofía y Literatura, v. 30, n. 1, p. 69-85, 2020.

DAISEY, P. The reading experiences and beliefs of secondary pre-service. Reading Horizons, v. 49, n. 2, p. 69-85, 2009.

DECI, E. L.; RYAN, R. M. Intrinsic motivation and self-determination in human behavior. Nueva York: Plenum: Springer, 1985.

DELBRAYELLE, A.; DUSZYNSKI, M. De la difficulté à se construire comme un sujet lecteur didactique quand on est professeur des écoles stagiaire. In: LEBRUN, A. M.; ROUXEL, A.; VARGAS, C. (ed.). La littérature et l'école: enjeuX, resistances, perspectives. Aix-en-Provence: Universitaires de Provence, 2007. p. 101-111.

DOMÍNGUEZ-PÉREZ, D. A.; PÉREZ-RUL, M. N. Internet y el hábito de enseñar en la universidad. Innovación Educativa, Ciudad de México, v. 9, n. 49, p. 11-17, 2009.

ELCHE, M.; YUBERO, S. La lista completa de docentes con conferencias: el comportamiento del docente de Educación Infantil y Educación Primaria. Bordón, Madrid, v. 71, n. 1, p. 31-45, 2019.

FELIPE, A.; BARRIOS, E. Evaluación de la competencia docente de futuros docentes. Investigaciones sobre Lectura, Málaga, v. 7, p. 7-21, 2017.

FELIPE, A.; VILLANUEVA, J. D. Diseño y validación de un proyecto piloto para la evaluación de la competencia académica de estudiantes universitarios. Investigaciones sobre Lectura, Málaga, v. 10, p. 95-117, 2018.

GRANADO, C.; PUIG, M. ¿Qué pasa con los futuros maestros y maestros y maestros? Un estudio docente como asignatura a través de los títulos que evocan. Ocnos, Ciudad Real, v. 11, n. 1, p. 93-112, 2014. 
JARPA, M. Escritura académica para el desarrollo de la reflexión pedagógica sobre la formación del profesorado: la crónica del profesor-director. Íkala: Revista de Lenguaje y Cultura, Medellín, v. 24, n. 1, p. 85-101, 2019.

JARPA, M.; BECERRA, N. Escritura para la reflexión pedagógica: concepciones y géneros discursivos que escriben los estudiantes en dos carreras de pedagogía. Logos: Revista de Lingüística, Filosofía y Literatura, La Serena, v. 29, n. 2, p. 364-381, 2019.

JIMÉNEZ-PÉREZ, E. La inteligencia emocional como predictor del hábito de lectura y la competencia lectora en estudiantes universitarios. Investigaciones sobre Lectura, Málaga, v. 10, p. 30-54, 2018.

JUÁREZ, M. Influencia de la formación inicial del profesor sobre los hábitos de enseñanza y sobre el concepto de educación literaria. Investigaciones sobre Lectura, Málaga, v. 12, p. 99-115, 2019.

LARRAÑAGA, E.; YUBERO, S. Estudio sobre los hábitos de enseñanza de los universitarios españoles. Madrid: Fundación SM, 2008.

LOMAS, C. Leer para comprender y transformar el mundo. Enunciación, Bogotá, v. 8, n. 1, p. 57-67, 2003.

MATAS, A. Diseño del formato de escala Likert: el estado de la encuesta. Revista Electrónica de Investigación Educativa, Ensenada, v. 20, n. 1, p. 38-47, 2018.

MENDOZA, A. La investigación didáctica de las primeras lenguas. Educatio Siglo XXI, Murcia, v. 29, n. 1, p. 31-80, 2011.

MUNITA, F. El sujeto lector didáctico: "lectores que enseñan y profesores que leen". Álabe, Almería, v. 17, p. 29-48, 2018.

MUNITA, F. Reading habits of pre-service teachers. Culture and Education, v. 26, n. 3, p. 448-475, 2014.

MUNITA, F. "Volver a la lectura", o la importancia de la lectura personal en la formación continua del profesorado en didáctica de la lengua y la literatura. Profesorado: revisión del currículum y formación del profesorado, Granada, v. 23, n. 3, p. 413-430, 2019.

MUÑOZ, C. et al. Características psicométricas de una escala para caracterizar la motivación por la lectura académica. Revista Electrónica de Investigación Educativa, Ensenada, v. 14, n. 2, p. 118-132, 2012.

MUÑOZ, C. et al. Mejora en la motivación por la lectura académica: la mirada de estudiantes motivados. Ocnos, Ciudad Real, v. 15, n. 1, p. 52-68, 2016. 
MUÑOZ, C. et al. Profesores que leen y enseñan a leer: el lugar de la lectura durante la formación. Revista Espacios, Caracas, v. 39, n. 40, p. 32-43, 2018.

ORIOL-GRANADO, X. et al. Emociones positivas, apoyo a la autonomía y rendimiento de estudiantes universitarios: el papel mediador del compromiso académico y la autoeficacia. Revista de Psicodidáctica, País Vasco, v. 22, n. 1, p. 45-53, 2017.

PARODI, G. et al. Generación Google o generación Gutenberg: hábitos y propósitos de lectura en estudiantes universitarios chilenos. Comunicar, v. 27, n. 58, p. 85-94, 2019.

PARRADO, M.; ROMERO, M. F.; TRIGO, E. La experiencia literaria en la formación de futuros docentes: el viaje iniciático de nuestras biografías en 10 hashtag. In: AMAR RODRÍGUEZ, V. (org.). Miradas y voces de futuros maestros. Barcelona: Octaedro, 2018. p. 59-86.

PEREDO, M. A. Las habilidades de la lectura y la escolaridad. Perfiles Educativos, Ciuad de México, v. 23, n. 94, p. 57-69, 2001.

PÉREZ-PEITX, M. El pensament sobre l'ensenyament i l'aprenentatge de la lectura i l'escriptura durant la formació inicial dels Mestres. Llengua, Societat i Comunicació, Barcelona, v. 14, p. 82-89, 2016.

PLAZAOLA, I.; RUIZ, U.; IRIONDO, I. Análisis de la propia actividad en el Practicum: una experiencia formativa. Revista Complutense de Educación, Madrid, v. 29, n. 4, p. 33-52, 2018.

ROMERO, M. F.; JIMÉNEZ, R. EI Practicum del MAES y la formación inicial en la enseñanza de lenguas: entre la realidad y el deseo. Lenguaje y Textos, Valencia, v. 39, p. 49-58, 2014.

ROMERO, M. F.; JIMÉNEZ, R. La escritura académica como estrategia de centro en la Facultad de Ciencias de la Educación de Cádiz. In: BALLANO, I.; MUÑOZ, I. (coord.). La escritura académica en las universidades españolas. Bilbao: DeustoDigital, 2015. p. 6394.

ROMERO, M. F.; SALVADOR, A.; TRIGO, E. Propuestas para trabajar la escritura académica en los estudios universitarios. In: ROMERO, M. F. (coord.). La escritura académica: diagnóstico y propuestas de actuación. Barcelona: Octaedro, 2014. p. 87100.

ROMERO, M. F.; TRIGO, E. Herramientas para el éxito. Cuadernos de Pedagogía, Barcelona, n. 458, p. 16-21, 2015.

SÁEZ, J. M. Investigación educativa. Fundamentos teóricos, procesos y elementos prácticos (enfoque práctico con ejemplos. Esencial para TFG, TFM y tesis). UNED, 2017. 
SÁNCHEZ-JIMÉNEZ, M. Á.; GALIANO, A. Desarrollo de un equipo docente en la coordinación del profesorado en el ámbito universitario. Alteridad: Revista de Educación, Quito, v. 14, n. 1, p. 98-108, 2019.

SANTOS-DÍAZ, I. C. Incidencia de la lectura en el vocabulario en lengua materna y extranjera. Ocnos, Ciudad Real, v. 16, n. 1, p. 79-88, 2017.

SANTOS-DÍAZ, I. C.; TRIGO, E.; ROMERO, M. F. La exposición informal a una lengua extranjera (inglés y francés) y la competencia léxica. Language for International Communication: Linking Interdisciplinary Perspectives, Latvia, p. 431-439, 2020.

SILVIA, P. J. Exploring the psychology of interest. Oxford: Oxford University, 2006.

TERCANLIOGLU, L. Pre-service teachers as readers and future teachers of EFL reading. TESL-EJ, v. 5, n. 3, p. 1-17, 2001.

TRIGO, E.; NÚÑ̃Z, X. Análisis competencial de la escritura académica en Español Lengua Extranjera (ELE) de estudiantes portugueses. Aula de Encuentro, Jaén, v. 20, n. 2, p. 116-139, 2018.

WIGFIELD, A.; ECCLES, J. Expectancy-Value Theory of Achievement Motivation. Contemporary Educational Psychology, n. 25, p. 68-8, 2000.

YOON, H.-J.; RÖMER, U. Quantifying Disciplinary Voices: An Automated Approach to Interactional Metadiscourse in Successful Student Writing. Written Communication, v. 37, n. 2, p. 1-37, 2020.

\section{Anexo}

Tabla 1 - Resultados de la Escala de Motivación pro la Lectura Académica (EMLA)

\begin{tabular}{|c|c|c|c|c|c|c|c|}
\hline & Escala Motivacional de Lectura Académica (EMLA) & & 1 & 2 & 3 & 4 & 5 \\
\hline \multirow{2}{*}{1} & \multirow{2}{*}{$\begin{array}{l}\text { La lectura de textos académicos es útil para mi } \\
\text { formación profesional. }\end{array}$} & v & 6 & 14 & 39 & 45 & 50 \\
\hline & & $\%$ & 3,90 & 9,09 & 25,32 & 29,22 & 32,47 \\
\hline \multirow{2}{*}{2} & \multirow{2}{*}{$\begin{array}{c}\begin{array}{c}\text { Es importante leer toda la bibliografía mínima de los } \\
\text { cursos. }\end{array} \\
\text {. }\end{array}$} & $\mathrm{N}$ & 32 & 41 & 47 & 26 & 8 \\
\hline & & $\%$ & 20,78 & 26,62 & 30,52 & 16,88 & 5,19 \\
\hline \multirow{2}{*}{3} & \multirow{2}{*}{$\begin{array}{l}\text { Me gusta leer textos académicos relacionados con } \\
\text { mi carrera. }\end{array}$} & $\mathrm{N}$ & 22 & 23 & 46 & 47 & 16 \\
\hline & & & 14,29 & 14,94 & 29,87 & 30,52 & 10,39 \\
\hline \multirow[b]{2}{*}{4} & \multirow{2}{*}{$\begin{array}{c}\text { Soy capaz de dejar otros intereses de lado y } \\
\text { comprometerme hasta terminar de manera debida } \\
\text { la lectura de un texto. }\end{array}$} & 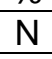 & 18 & 35 & 39 & 39 & 23 \\
\hline & & $\%$ & 11,69 & 22,73 & 25,32 & 25,32 & 14,94 \\
\hline \multirow{2}{*}{5} & \multirow{2}{*}{$\begin{array}{c}\text { Considero que escojo bien los textos académicos } \\
\text { que me ayudan a realizar mis trabajos. }\end{array}$} & $\mathrm{N}$ & 8 & 26 & 69 & 37 & 14 \\
\hline & & & 5,19 & 16,88 & 44,81 & 24,03 & 9,09 \\
\hline \multirow{2}{*}{6} & \multirow{2}{*}{$\begin{array}{l}\text { Considero de gran utilidad entender los textos } \\
\text { académicos que se me asignan. }\end{array}$} & & 7 & 14 & 4 & 50 & 40 \\
\hline & & 70 & 4,55 & 9,09 & 27,92 & 32,47 & 25,97 \\
\hline \multirow{2}{*}{7} & \multirow{2}{*}{$\begin{array}{c}\text { Para mí, la bibliografía mínima es importante para } \\
\text { comprender la materia de los cursos. }\end{array}$} & $\mathrm{N}$ & 29 & 35 & 57 & 27 & 6 \\
\hline & & $\%$ & 18,83 & 22,73 & 37,01 & 17,53 & 3,90 \\
\hline \multirow[b]{2}{*}{8} & \multirow{2}{*}{$\begin{array}{l}\text { El tiempo que utilizo en leer textos académicos, } \\
\text { implica dejar de hacer otras cosas. }\end{array}$} & $\mathrm{N}$ & 5 & 13 & 39 & 46 & 51 \\
\hline & & $\%$ & 3,25 & 8,44 & 25,32 & 29,87 & 33,12 \\
\hline
\end{tabular}

Educ. Form., Fortaleza, v. 6, n. 1, e3535, jan./abr. 2021

DOI: https://doi.org/10.25053/redufor.v6i1.3535

https://revistas.uece.br/index.php/redufor/index 
Tabla 1 - Resultados de la Escala de Motivación pro la Lectura Académica (EMLA)

(conclusión)

\begin{tabular}{|c|c|c|c|c|c|c|c|}
\hline & \multirow{2}{*}{ Escala Motivacional de Lectura Académica (EMLA) } & & & & & & \\
\hline & & & 1 & 2 & 3 & 4 & 5 \\
\hline \multirow{2}{*}{9} & \multirow{2}{*}{$\begin{array}{l}\text { Al leer un texto académico, logro captar las ideas } \\
\text { centrales. }\end{array}$} & $\mathrm{N}$ & 2 & 11 & 49 & 58 & 34 \\
\hline & & $\%$ & 1,30 & 7,14 & 31,82 & 37,66 & 22,08 \\
\hline \multirow{2}{*}{10} & \multirow{2}{*}{$\begin{array}{c}\text { Me interesa leer material más allá de lo requerido por } \\
\text { el curso. }\end{array}$} & $\mathrm{N}$ & 23 & 38 & 51 & 32 & 10 \\
\hline & & $\%$ & 14,94 & 24,68 & 33,12 & 20,78 & 6,49 \\
\hline \multirow{2}{*}{11} & \multirow{2}{*}{$\begin{array}{l}\text { Considero útil la lectura de textos académicos para } \\
\text { desarrollar ciertas competencias profesionales. }\end{array}$} & $\mathrm{N}$ & 10 & 15 & 43 & 57 & 29 \\
\hline & & $\%$ & 6,49 & 9,74 & 27,92 & 37,01 & 18,83 \\
\hline \multirow{2}{*}{12} & \multirow{2}{*}{$\begin{array}{l}\text { Para mí, la bibliografía mínima es importante para } \\
\text { complementar la materia de los cursos. }\end{array}$} & $\mathrm{N}$ & 23 & 32 & 58 & 33 & 8 \\
\hline & & $\%$ & 14,94 & 20,78 & 37,66 & 21,43 & 5,19 \\
\hline \multirow{2}{*}{13} & \multirow{2}{*}{$\begin{array}{l}\text { Soy capaz de distinguir las ideas centrales de las } \\
\text { complementarias en los textos académicos. }\end{array}$} & $\mathrm{N}$ & 4 & 13 & 49 & 52 & 36 \\
\hline & & $\%$ & 2,60 & 8,44 & 31,82 & 33,77 & 23,38 \\
\hline \multirow{2}{*}{14} & \multirow{2}{*}{$\begin{array}{l}\text { Los textos académicos son un apoyo fundamental a } \\
\text { mi formación universitaria. }\end{array}$} & $\mathrm{N}$ & 14 & 21 & 46 & 51 & 22 \\
\hline & & $\%$ & 9,09 & 13,64 & 29,87 & 33,12 & 14,29 \\
\hline \multirow{2}{*}{15} & \multirow{2}{*}{$\begin{array}{c}\text { Me interesan textos de disciplinas asociadas a mi } \\
\text { área de estudio. }\end{array}$} & $\mathrm{N}$ & 7 & 24 & 52 & 44 & 27 \\
\hline & & $\%$ & 4,55 & 15,58 & 33,77 & 28,57 & 17,53 \\
\hline \multirow{2}{*}{16} & \multirow{2}{*}{$\begin{array}{c}\text { A pesar de que algunos textos académicos son } \\
\text { complejos, soy capaz de comprenderlos si me } \\
\text { esfuerzo. }\end{array}$} & $\mathrm{N}$ & 5 & 12 & 48 & 63 & 26 \\
\hline & & $\%$ & 3,25 & 7,79 & 31,17 & 40,91 & 16,88 \\
\hline \multirow{2}{*}{17} & \multirow{2}{*}{$\begin{array}{c}\text { La lectura de textos académicos me ayudará a ser } \\
\text { un buen profesional. }\end{array}$} & . & 7 & 2 & 7 & 52 & 29 \\
\hline & & $\%$ & 4,55 & 14,29 & 28,57 & 33,77 & 18,83 \\
\hline \multirow{2}{*}{18} & \multirow{2}{*}{$\begin{array}{l}\text { Considero importante la lectura complementaria } \\
\text { que sugieren los programas de los cursos. }\end{array}$} & $\mathrm{N}$ & 15 & 33 & 56 & 43 & 7 \\
\hline & & $\%$ & 9,74 & 21,43 & 36,36 & 27,92 & 4,55 \\
\hline \multirow{2}{*}{19} & \multirow{2}{*}{$\begin{array}{l}\text { Leo textos por gusto y soy capaz de leer otros no } \\
\text { obligatorios. }\end{array}$} & $\mathrm{N}$ & 22 & 35 & 38 & 36 & 23 \\
\hline & & $\%$ & 14,29 & 22,73 & 24,68 & 23,38 & 14,94 \\
\hline \multirow[b]{2}{*}{20} & & $\mathrm{~N}$ & 11 & 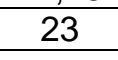 & 3 & 56 & 27 \\
\hline & $\begin{array}{c}\text { dedico todo el tiempo necesario hasta t } \\
\text { manera adecuada. }\end{array}$ & $\%$ & 7,14 & 14,94 & 24,03 & 36,36 & 17,53 \\
\hline 21 & Estoy capacitado para leer la I & $\mathrm{N}$ & 4 & & 4 & 59 & 37 \\
\hline & & $\%$ & 2,60 & 8,44 & 26,62 & 38,31 & 24,03 \\
\hline & Cuando tengo dudas des & $\mathrm{N}$ & 26 & 47 & 41 & 29 & 11 \\
\hline 22 & & $\%$ & 16,88 & 30,52 & 26,62 & 18,83 & 7,14 \\
\hline & Para mí, es importante comprender & $\mathrm{N}$ & 13 & 47 & 45 & 38 & 11 \\
\hline 23 & dominar cie & $\%$ & 8,44 & 30,52 & 29,22 & 24,68 & 7,14 \\
\hline 24 & $\mathrm{~A}$ & $\mathrm{~N}$ & 9 & 21 & 49 & 50 & 25 \\
\hline 24 & Ios textos asoclade & $\%$ & 5,84 & 13,64 & 31,82 & 32,47 & 16,23 \\
\hline 25 & Para mí, es importante tener leída la bibliografía & $\mathrm{N}$ & 38 & 39 & 47 & 24 & 6 \\
\hline 25 & all & $\%$ & 24,68 & 25,32 & 30,52 & 15,58 & 3,90 \\
\hline 26 & $N$ & $\mathrm{~N}$ & 44 & 42 & 46 & 19 & 3 \\
\hline & & $\%$ & 28,57 & 27,27 & 29,87 & 12,34 & 1,95 \\
\hline 27 & & $\mathrm{~N}$ & 16 & 21 & 46 & 48 & 23 \\
\hline 27 & capaz de invertir más tiempo en su lectura. & $\%$ & 10,39 & 13,64 & 29,87 & 31,17 & 14,94 \\
\hline
\end{tabular}

Educ. Form., Fortaleza, v. 6, n. 1, e3535, jan./abr. 2021

DOI: https://doi.org/10.25053/redufor.v6i1.3535

https://revistas.uece.br/index.php/redufor/index 
Inmaculada Clotilde Santos Díaz, Universidad de Málaga, Departamento de Didáctica del Lenguaje, Artes y Deportes

iDhttps://orcid.org/0000-0002-0066-7783

Doctora en Innovación Educativa por la Universidad de Málaga. Profesora ayudante doctora del Departamento de Didáctica de la Lengua, las Artes y el Deporte de la Universidad de Málaga, coordinadora provincial de Lectura Escolar y Biblioteca de la Delegación Territorial de Educación de Málaga y experta evaluadora y controladora de proyectos Erasmus para el Servicio Español de Internacionalización de la Educación. Su investigación, trasladada a las revistas $J C R$ y $S J R$, se centra en la lingüística aplicada a la enseñanza de idiomas, donde tiene una amplia formación. Actualmente forma parte de dos proyectos europeos y es la coordinadora académica de las movilidades KA203 con China. También ha participado en numerosos congresos internacionales.

Contribución de autoría: Análisis y procesamiento de los datos.

E-mail:santosdiaz@uma.es

\section{María Juárez Calvillo, Universidad de Málaga}

iiiohttps://orcid.org/0000-0002-5767-9013

Licenciada en Filología Hispánica y Inglesa por la Universidad de Cádiz. Realizó el máster en Estudios Ingleses y Comunicación Intercultural en la Universidad de Málaga y el máster en Docencia en la especialidad de Inglés en la Universidad de Cádiz. Es estudiante de doctorado en la Universidad de Málaga y su tesis trata sobre la influencia de los hábitos de lectura y el consumo audiovisual de los futuros profesores de educación infantil y primaria de la Universidad de Cádiz en su disponibilidad léxica multilingüe, un caso de estudio de la Universidad de Cádiz. Publicó en revistas como ISL y Dykinson.

Contribución de autoría: Recogida de los datos y comentario de los resultados.

E-mail: maria.juarezcalvillo@gmail.com

\section{Ester Trigo Ibáñez, Universidad de Cádiz, Departamento de Didáctica de la Lengua y la Literatura} iii (i) http://orcid.org/0000-0003-3035-4398

Doctora en Lingüística por la Universidad de Cádiz. Profesora contratada doctora del Departamento de Didáctica de la Lengua y la Literatura en la Universidad de Cádiz. Sus líneas de investigación son diversas: escritura académica, de la que ha publicado varios capítulos en altos niveles de SPI; educación literaria, desde donde dirigió una tesis doctoral y publicó varios artículos de SJR; disponibilidad léxica, cuya tesis doctoral forma parte del Proyecto Léxico de Disponibilidad Panhispánica y se enfoca en proyectos de idiomas. Sus publicaciones son el resultado de: participación en proyectos de investigación, acciones avaladas para la mejora de la docencia, tesis doctorales focalizadas, tesis o investigaciones propias en Polonia, Chile y Portugal.

Contribución de autoría: Revisión teórica.

E-mail: ester.trigo@uca.es

Editora responsable: Lia Machado Fiuza Fialho

Pareceristas ad hoc: María Victoria Mateo García y María Natalia Castillo Fadic

\section{Cómo citar este artículo (ABNT):}

SANTOS DÍAZ, Inmaculada Clotilde; JUÁREZ CALVILLO, María; TRIGO IBÁÑEZ, Ester. Motivación por la lectura académica de futuros docentes. Educ. Form., Fortaleza, v. 6, n. 1, e3535, 2020. Disponible en: https://revistas.uece.br/index.php/redufor/article/view/3535

Educ. Form., Fortaleza, v. 6, n. 1, e3535, jan./abr. 2021

DOI: https://doi.org/10.25053/redufor.v6i1.3535

https://revistas.uece.br/index.php/redufor/index 


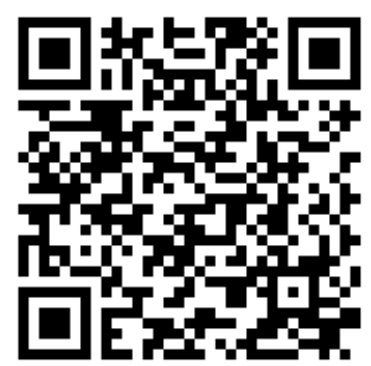

Recibido el 13 de julio de 2020.

Aceptado el 4 de agosto de 2020.

Publicado el 20 de ocutubre de 2020. 